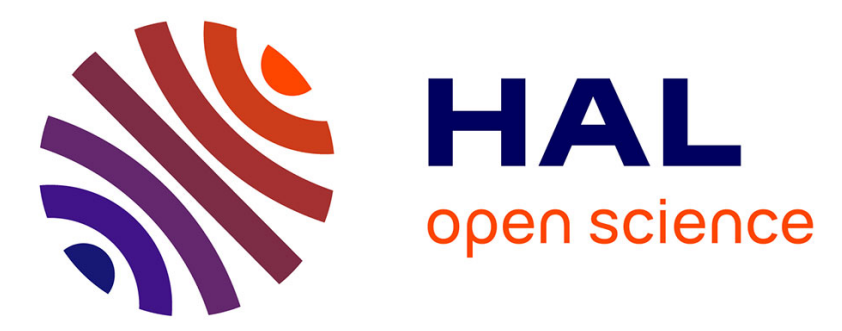

\title{
Vibrational modes of aminothiophenol: a TERS and DFT study
}

\author{
Alexandre Merlen, M. Chaigneau, Stéphane Coussan
}

\section{To cite this version:}

Alexandre Merlen, M. Chaigneau, Stéphane Coussan. Vibrational modes of aminothiophenol: a TERS and DFT study. Physical Chemistry Chemical Physics, 2015, 17 (29), pp.19134-19138. $10.1039 / \mathrm{c} 5 \mathrm{cp} 01579 \mathrm{k}$. hal-02266146

\section{HAL Id: hal-02266146 https://hal.science/hal-02266146}

Submitted on 13 Aug 2019

HAL is a multi-disciplinary open access archive for the deposit and dissemination of scientific research documents, whether they are published or not. The documents may come from teaching and research institutions in France or abroad, or from public or private research centers.
L'archive ouverte pluridisciplinaire HAL, est destinée au dépôt et à la diffusion de documents scientifiques de niveau recherche, publiés ou non, émanant des établissements d'enseignement et de recherche français ou étrangers, des laboratoires publics ou privés. 


\title{
Vibrational modes of aminothiophenol : a TERS and DFT study
}

\author{
A. Merlen ${ }^{1 *}$, M. Chaigneau ${ }^{2}$, S. Coussan ${ }^{3}$ \\ 1 IM2NP, UMR-CNRS 7334, Site de l'Université de Toulon, 83957 La Garde Cedex, France. \\ 2 LPICM, UMR-CNRS 7647, Ecole Polytechnique, Palaiseau, France. \\ 3 Laboratoire Physique des Interactions Ioniques et Moléculaires, UMR 7345-CNRS, \\ Aix-Marseille Université, Centre St-Jérôme, 13397 Marseille Cedex 20, France. \\ * Corresponding author: merlen@univ-tln.fr \\ Submitted to PCCP
}

\begin{abstract}
We report Tip Enhanced Raman Spectroscopy (TERS) mapping and Density Functional (DFT) calculations of aminothiophenol (ATP) grafted on a gold surface. The TERS mapping has demonstrated Raman modes of (ATP) and its dimerised derivative Dimercaptoazobenzene (DMAB). This feature confirms that the plasmon activated chemical reaction of ATP has occurred during TERS measurements. In some specific part of the samples some unidentified Raman modes are observed. We suggest that they could come from intermediate species formed during the conversion of ATP into DMAB. These modes are compared with calculated Raman spectra of some possible intermediate species. These results confirm the high potentiality of TERS measurements for nanochemistry.
\end{abstract}

\section{Introduction}

Metallic nanoparticles have already demonstrated their huge potentiality both in chemistry and physics. Based on collective electronic oscillation, their plasmonic properties can be used for highly sensitive optical spectroscopy ${ }^{1,2}$, photo thermal therapy ${ }^{3}$, photovoltaics ${ }^{4}$... Recently a new field has emerged: Plasmon driven surface catalyzed reactions ${ }^{5}$. For some specific molecules linked to metallic nanoparticles (mainly gold or silver, these two metals exhibit strong plasmonic properties in the visible range), it has been shown by Surface Enhanced Raman Spectroscopy (SERS ${ }^{6}$ ) measurements that a chemical reaction could occur when the plasmonic resonance of the nanoparticle is excited by an incoming light. In this case the localized plasmon act as a catalyst to activate the reaction. Such mechanism has been reported for Aminothiophenol (ATP, also known as Aminobenzenethiol or Paramercaptoaniline) and Nitrothiophenol (NTP). During laser illumination, new vibrational modes are observed in the Raman spectra of these molecules. These modes have been first associated to a charge transfer mechanism ${ }^{7}$ (associated to the chemical effect in the enhancement mechanism of SERS) or discussed in terms of change in the molecular orientation ${ }^{8}$. Nevertheless some recent studies have demonstrated that they are in fact vibrational modes of Dimercaptoazobenzene (DMAB) ${ }^{9}$ : two ATP (or NTP) molecules react on the metallic surface to form this new molecule. Many SERS studies have since confirmed this hypothesis. A mechanism to explain this transformation has even been proposed. Nevertheless there is still a debate in the SERS community and new techniques are necessary to confirm this hypothesis. A promising tool is Tip Enhanced Raman Spectroscopy $\left(\right.$ TERS $\left.^{10}\right)$. This technique, similar to SERS, is based on the giant electromagnetic enhancement present at the apex of a metallic nanoantenna ${ }^{11}$. This electromagnetic enhancement is used to amplify the Raman signal. SERS requires a network of nanoantennas and the molecules are usually deposited on 
this network. As a consequence SERS must be performed with specific substrates, which is a major limitation of this technique. In opposition, TERS can be performed with any kind of sample: the unique nanoantenna (usually a metallic tip) is simply approached to the sample thanks to a scanning probe microscope. The recorded Raman signal comes from the molecules localized a few nanometers around the apex of the tip. It is thus possible to have a high sensitivity associated to a nanometric resolution, similar to what can be obtained with optical near field devices ${ }^{12}$. TERS appears thus as a perfect tool to study the chemical transformation of ATP or NTP into DMAB and several studies have reported such measurements. They have confirmed the high reactivity of ATP or NTP. Lantman et al. have observed temporal fluctuations in the TERS signal of NTP ${ }^{13}$. Performing high vacuum TERS measurements Sun et al. have observed some unidentified vibrational modes ${ }^{14}$. Nevertheless none of these studies has tried to take advantage of the high spatial resolution of TERS to perform mapping. In this article, we report such mapping performed on ATP deposited on a gold annealed sample. On some specific part of the sample new Raman modes are observed. These modes are compared to DFT calculations. We suggest that they could come from intermediate species formed during the transformation of ATP into DMAB.

\section{Method}

A SAM layer of ATP was prepared following the usual protocol ${ }^{15}$. The atomically flat $A u(111)$ substrates were purchased from Arrandee. A $1 \mathrm{mM}$ solution of ATP molecules in ethanol was used for the SAM absorption on the freshly flame annealed substrate during an absorption time of $12 \mathrm{~h}$. A Flat flame annealed surface was used to avoid any strong SERS background signal that could arise from gold nanostructures.

Our TERS set-up consists of a confocal Raman microscope (Labram HR800, HORIBA Jobin Yvon) with a $633 \mathrm{~nm}$ HeNe laser source optically coupled in an oblique backscattering geometry to a scanning probe microscope (XE-100, Park Systems). The $633 \mathrm{~nm}$ excitation is far from the absorption bands of ATP ${ }^{16}$ and resonant mechanism are excluded in our measurements. The long-working-distance objective ( $50 \times, N A=0.45)$ is oriented at $65^{\circ}$ with respect to the sample normal (figure 1$)$. The polarization state of the incident beam is p-polarized (electric field parallel to the scattering plane). Further details about the experimental setup can be found in other references ${ }^{17-19}$. The SPM is operated in scanning tunnelling microscopy (STM) mode; the tips used were prepared from Au wire pieces of a $250 \mu \mathrm{m}$ diameter and $99.99 \%$ purity (from Goodfellow) by electrochemical etching in a concentrated $\mathrm{HCl}$ / ethanol 1:1 solution. It must be said that a recent study has shown that the STM voltage has not much influence in the TERS measurements of ATP molecules ${ }^{14}$. The TERS mapping was performed over a $0.8 \times 0.8 \mu \mathrm{m}^{2}$ area (number of pixels $20 \times 20$ ) by recording a Raman spectrum at every $40 \mathrm{~nm}$ with a $1 \mathrm{~s}$ acquisition time. These experimental parameters offers a good compromise between total acquisition time, spatial resolution, high quality Raman spectra and total scan size.

The calculations of Raman modes were performed within Density Functional Theory (DFT) using the GAUSSIAN 09 suite $^{20}$. We used the PW91PW91 functional ${ }^{21}$, a 6-311+G(2d,p) basis set for $\mathrm{C}, \mathrm{N}, \mathrm{S}$ and $\mathrm{H}$ and a LANDL2DZ basis set for $\mathrm{Au}^{22}$. Those parameters are known to be efficient for the calculations of ATP and DMAB on gold ${ }^{23}$. 
FDTD simulations were performed using Lumerical software ${ }^{24}$ with parameters that have already been used for others simulations ${ }^{25,26}$.

\section{Results and Discussion}

A typical STM image of the gold surface can be seen in figure 1c. It presents smooth and flat areas separated by a grain boundary. Such a surface is typical of what can be obtained with annealed gold. The average intensity of the TERS signal for the same surface is shown in figure $1 \mathrm{~d}$. A good correlation between the TERS signal and the topography is observed. We can estimate the optical resolution to be lower than $100 \mathrm{~nm}$, a much better resolution than what can be obtained with classical optical Raman microscopy. This confirms that the Raman signal really comes from the limited area below the tip as can be seen in figure1b. In addition no far-field Raman signal is observed when the tip is removed from the surface. A higher resolution could be obtained using a smaller mapping step size at the cost of a much longer total time acquisition. The TERS signal intensity is too low (ranging from 100 to $1000 \mathrm{cts} / \mathrm{sec}$ ) to ensure a reasonable total time acquisition of the mapping compatible with a limited drift of the piezo-table.

a)

b)

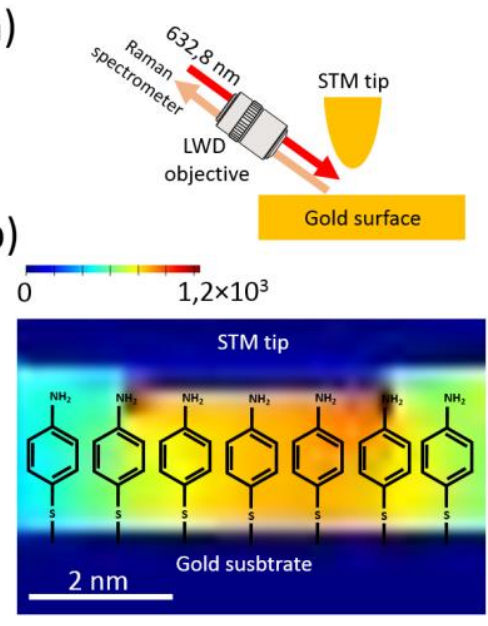

c)

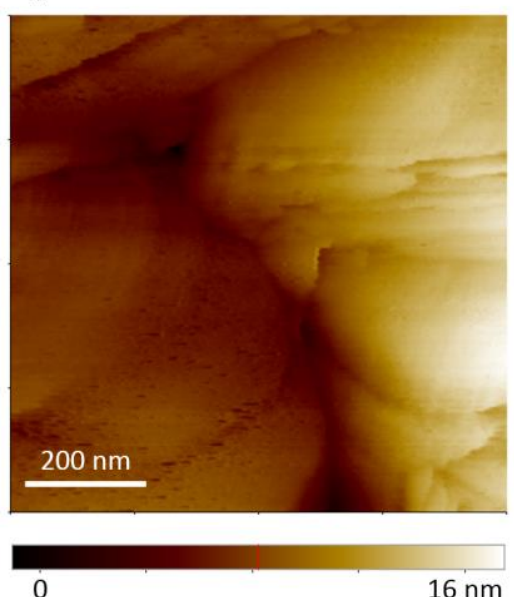

d)

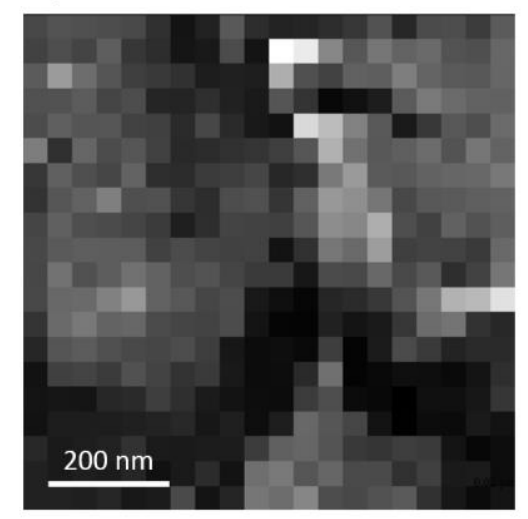

Figure 1: a) Experimental setup configuration b) Distribution of the enhanced electromagnetic field between the tip and the gold surface (FDTD calculations) c) STM image of the gold surface ( $800 \times 800$ $\mathrm{nm}^{2}$ ) d) Corresponding TERS mapping of the same surface (average intensity integrated between 1500 and $\left.1620 \mathrm{~cm}^{-1}\right)$.

Some typical TERS spectra are reported (and the corresponding location are pointed by arrows on the TERS mapping) in figure 2. Most of the Raman spectra exhibit 2 peaks located at 1080 and 1580 $\mathrm{cm}^{-1}$. These two modes are associated to ATP molecules, this is clearly confirmed by DFT simulations of the ATP Raman modes (figure $2 \mathrm{c}$ ). In some particular places of the sample, other modes are observed: 1140, 1390 and $1440 \mathrm{~cm}^{-1}$ (see fig. 2 (b), TERS spectrum in the middle). These modes have already been reported in many SERS studies of ATP and have led to many debates. As mentioned in the introduction, those Raman peaks has been first attributed to a charge transfer mechanism ${ }^{27,28}$. Nevertheless, some recent studies have demonstrated that their presence strongly depends on several experimental parameters: density of the ATP layer, laser power, etc... ${ }^{15}$ These features are hardly compatible with a charge transfer mechanism and based on DFT calculations several authors 
have proposed to attribute them to a new molecule: $D M A B^{9,29-31}$. This molecule should be formed via dimerization of ATP molecules activated by an electromagnetic and plasmonic process occurring under the tip. Such a reaction can also be observed with nitrothiophenol molecules ${ }^{5}$.

a)

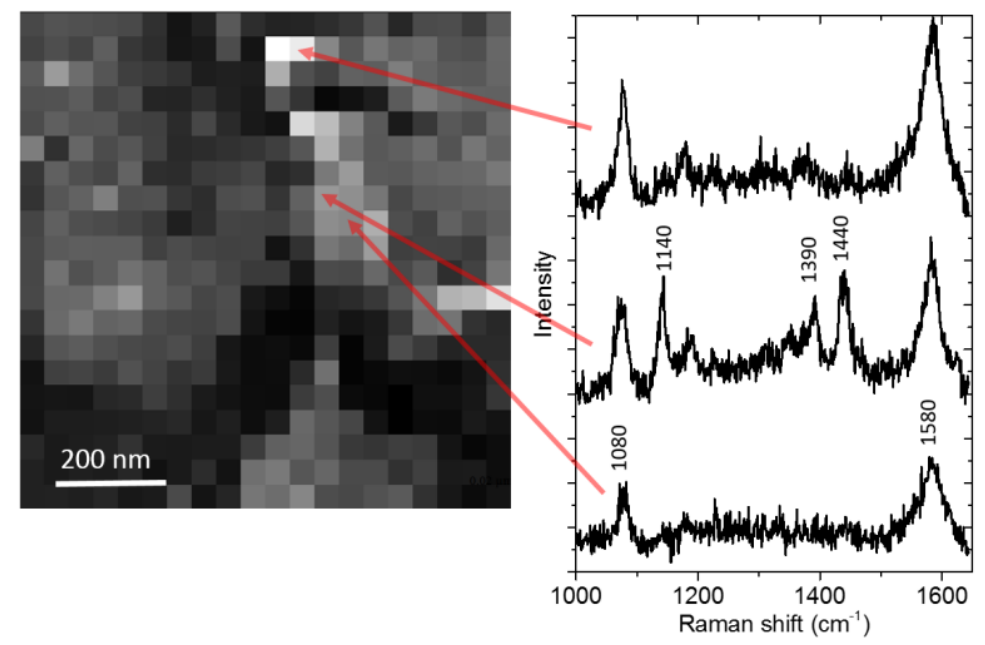

c)

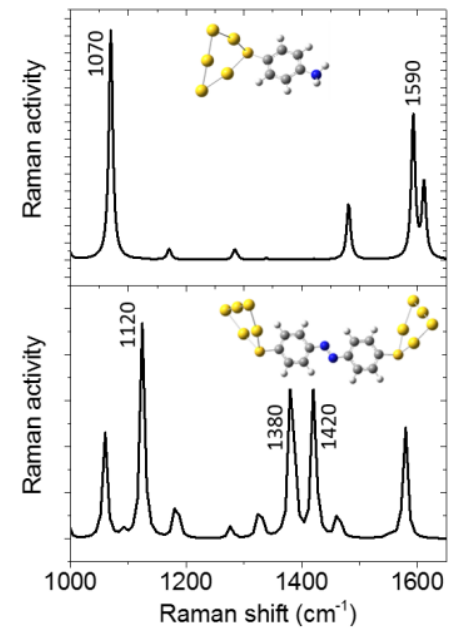

Figure 2: a) TERS mapping b) Raman spectra for different positions of the mapping. c) DFT calculations of the Raman modes for ATP (above) and DMAB (below). Both molecules are in interaction with a 5 atoms gold cluster.

Many experimental studies have been performed and it appears in the Raman community that this hypothesis is now privileged to explain the presence of these new modes $(1120,1380$ and $1420 \mathrm{~cm}$ $\left.{ }^{1}\right)$.

Based on these TERS mapping on ATP molecules grafted on a Au substrate, we can conclude that most of the layer on the gold surface is still ATP, and in random locations on the sample the Raman modes of DMAB are observed suggesting that the dimerization has occurred. Nevertheless, it appears that our illumination condition is not powerful enough to induce an immediate and total transformation of ATP into DMAB. It must be said that no clear correlation between the places where $D M A B$ molecules have been formed and the corresponding topography of the sample has been observed. The proposed mechanism for the transformation of ATP into DMAB is based on the giant electromagnetic enhancement present in the vicinity of plasmonic nanostructures. This enhancement is highly localized, as can be seen in the figure $1 \mathrm{~b}$ showing this distribution between the tip and the gold surface thanks to FDTD calculations. For gold nanostructures the electromagnetic enhancement is usually much higher at their edge. The relation between electromagnetic enhancement and the plasmon driven chemical reaction has been evidenced and discussed recently ${ }^{32}$. The gold annealed surface used in our study presents atomic flat grains but some edges and kinks are anyway present (see STM image in figure 1c). DMAB molecules should preferentially be formed at these edges but the spatial resolution of the TERS mapping is not enough to confirm this hypothesis. To study the correlation between electromagnetic enhancement and DMAB synthesis we suggest to perform similar TERS measurements on a sample with well-defined metallic nanostructures (such as nanodots ${ }^{26}$ or nanotriangles ${ }^{33}$ ). Nevertheless performing TERS mapping with such plasmonic nanostructures would lead to a strong SERS background signal which 
will decrease the final spatial resolution. Performing such TERS measurements remains an experimental challenge.

a)

b)

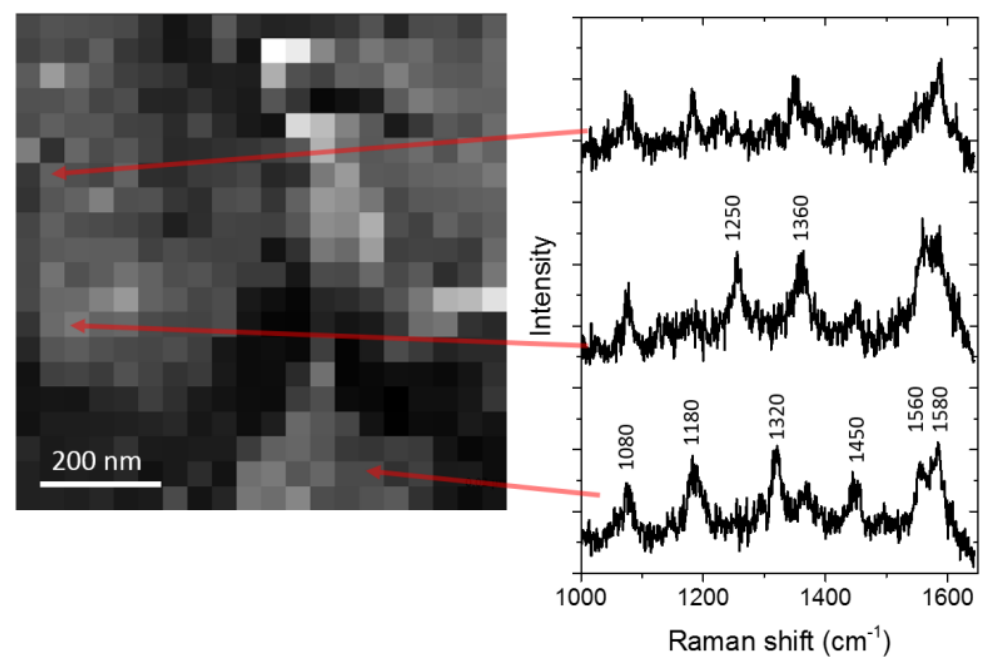

Figure 3: a) TERS mapping b) Others Raman spectra for different positions of the mapping indicated by the arrows.

In figure 3 are reported some Raman spectra that can neither be attributed to ATP or DMAB. These spectra are observed on a very limited number of places on the gold surface. This is the experimental reason why we have performed a rather large TERS scan at the cost of a lower spatial resolution: we wanted to observe a significant number of places where new Raman spectra are observed. Recently a high vacuum TERS measurements of ATP have reported the observation of new modes in the Raman spectrum of ATP ${ }^{14}$. The authors observed a considerably higher number of peaks compared to what we observed here. Some unidentified peaks were attributed to IR modes activated by the strong gradient-field effect ${ }^{34}$ at the tip apex. Even without performing any TERS mapping, the results shown in this article present a remarkably reproducible Raman signal. The fact that we have not observed such a high number of vibrational modes and that the other modes (corresponding to the $D M A B$ ) are observed only in some specific parts of the sample suggest that the unidentified modes presented in figure 3 should not be explained by a similar mechanism. Performing time resolved TERS measurements with nitrothiophenol, Lantman et al. $^{13}$ have observed intensity fluctuations and have associated them to a photoreaction, giving few suggestions about its exact nature. Recently, Zhao et al. ${ }^{35}$ have performed a DFT study about the chemical photoinduced reaction of azobenzene on nanostructured silver. For the conversion of ATP into DMAB, the authors have proposed the mechanism described in figure 4a. Even proposed for silver, one can reasonably assume that it would be the same on a gold surface: these two metals exhibit similar plasmonic properties in the visible range, except the fact that the silver resonance occurs at shorter wavelengths compared to gold. The proposed mechanism reaction for the conversion of ATP into DMAB is based on the formation of several intermediate species. Some are surely extremely reactive (the charged radical species) and can probably not be observed, but some others might be more stable. As already mentioned, our illumination condition is not powerful enough to induce an immediate and total transformation of 
ATP into DMAB. This suggests that some intermediate species could be observed in our TERS measurements and the induced vibrations associated to the unidentified Raman modes. To confirm this hypothesis, we have performed DFT calculations on some intermediate species (named DMHAB and DATP in figure 4a). It must be said that it is necessary to be cautious about the results given by these simulations: the mechanism of conversion is still under discussion ${ }^{36,37,29,38,39}$. A recent study of Xu et $a l .{ }^{40}$ has evidenced the role of oxygen and water in the conversion but the proposed intermediate species remain the same. We have also performed similar calculations with the free ATP molecule. Calculated Raman activities are reported in figure $4 \mathrm{~b}$ and $\mathrm{c}$.

a)
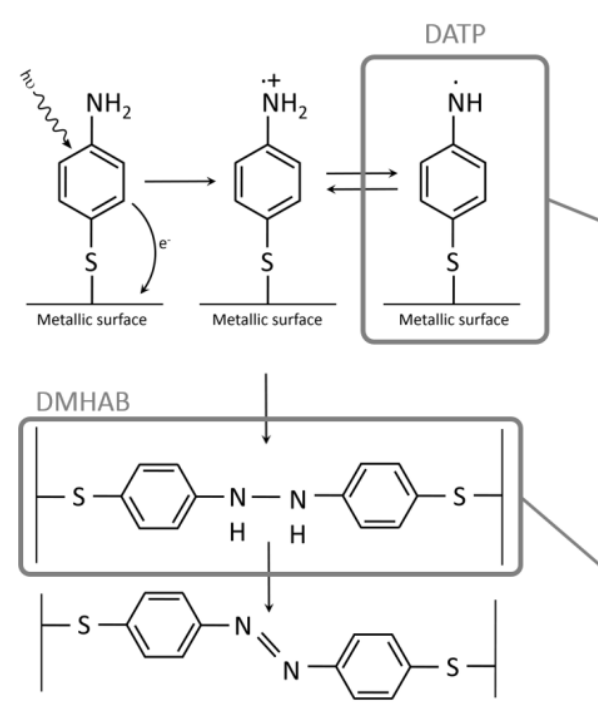

b)
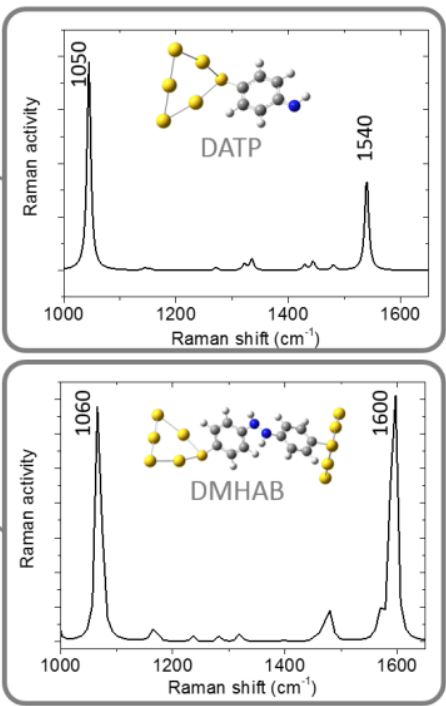

c)

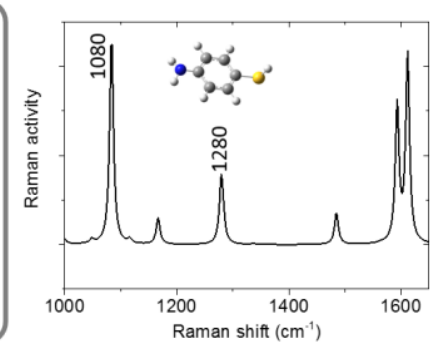

Figure 4: a) The chemical mechanism of synthesis of DMAB from ATP proposed by Zhao et al. ${ }^{35}$ b) DFT calculations of the Raman modes for different chemical intermediates DMAHB (for Dimercaptohydrazobenzene) and DATP (for deprotonated ATP) c) DFT calculations of a free ATP molecule.

For DATP, two main modes are observed: at 1050 and $1540 \mathrm{~cm}^{-1}$. Two main modes are also observed for DMHAB with slightly different positions: 1060 and $1600 \mathrm{~cm}^{-1}$. Our spectral resolution in TERS measurements is not enough to clearly distinguish two broad peaks separated by only $10 \mathrm{~cm}^{-1}$. As a consequence the observed mode at $1080 \mathrm{~cm}^{-1}$ cannot be used to distinguish some intermediate species. This is not the case for the higher frequency modes: in Raman spectra of figure 3 we can clearly distinguish a doublet at around $1600 \mathrm{~cm}^{-1}$, not observed in figure 2 . The lowest frequency mode of this doublet could be associated to DATP. In figure 3 there is also a broad mode observed in one spectrum at $1250 \mathrm{~cm}^{-1}$ and at $1320 \mathrm{~cm}^{-1}$ in the other. These peaks could be associated to the $1280 \mathrm{~cm}^{-1}$ mode reported for free ATP (see figure 4c), not observed when ATP is in interaction with gold. This hypothesis suggests that during the photochemical reaction some ATP molecules are desorbed from the gold surface. We also note that the relative intensity between all the calculated modes strongly depends on the considered specie. This could explain the intensity temporal fluctuations reported by Lantman et $a l^{13}$. As a conclusion, we suggest that these new modes could be the experimental fingerprint of intermediate species formed during the transformation of ATP into DMAB.

\section{Conclusion}


We have performed a TERS mapping on ATP molecules deposited on a flat gold surface. We have observed Raman modes of ATP and DMAB confirming that the plasmon driven dimerization can occur in such a configuration. We have also observed some unidentified vibrational modes. Our DFT calculations suggest that they could be the experimental fingerprints of intermediate species formed during the plasmon-driven dimerization. Our study confirms the huge potentiality of TERS for a better understanding of nanochemistry mechanisms.

Acknowledgements: The authors acknowledge Dr Nicolas Ferré, Dr Cédric Pardanaud and Pr Razvigor Ossikovski for fruitful discussions.

1 K. Kneipp, H. Kneipp, I. Itzkan, R. R. Dasari and M. S. Feld, Chem. Rev., 1999, 99, 2957-2975.

2 B. Pettinger, Mol. Phys., 2010, 108, 2039-2059.

3 X. Huang, P. K. Jain, I. H. El-Sayed and M. A. El-Sayed, lasers Med. Sci., 2009, 23, $217-228$

4 H. A. Atwater and A. Polman, Nat. Mater., 2010, 9, 205-13.

$5 \quad$ M. Sun and H. Xu, Small, 2012, 8, 2777-2786.

6 P. G. Etchegoin and E. C. Le Ru, Principles of surface-enhanced Raman spectroscopy and related plasmonic effects, Elsevier, 2009.

7 M. Osawa, N. Matsuda, K. Yoshii and I. Uchida, J. Phys. Chem., 1994, 98, $12702-$ 12707.

8 T. Chen, H. Wang, G. Chen, Y. Wang, Y. Feng, W. S. Teo, T. Wu and H. Chen, ACS Nano, 2010, 4, 3087-3094.

9 D. Y. Wu, X. M. Liu, Y. F. Huang, B. Ren, X. Xu and Z. Q. Tian, J. Phys. Chem. C, 2009, 113, 18212-18222.

10 R. M. Stockle, Y. D. Suh, V. Deckert and R. Zenobi, Chem. Phys. Lett., 2000, 318, 131.

11 A. Merlen and F. Lagugné Labarthet, Appl. Spectrosc., 2014, 68, 1307-1326.

12 A. Jorio and L. G. Cançado, Phys. Chem. Chem. Phys., 2012, 14, 15246-15256.

13 E. M. van S. Lantman, T. Deckert-Gaudig, A. J. G. Mank, V. Deckert and B. M. Weckhuysen, Nat. Nanotechnol., 2012, 7, 583-586.

14 M. Sun, Y. Fang, Z. Zhang and H. Xu, Phys. Rev. E. Stat. Nonlin. Soft Matter Phys., 2013, 87, 020401(R).

15 G. Delafosse, A. Merlen, S. Clair and L. Patrone, J. Chem. Phys., 2012, 136, 194704. 
16 A. Merlen, V. Gadenne, J. Romann, V. Chevallier, L. Patrone and J. C. Valmalette, Nanotechnology, 2009, 20, 7.

17 G. Picardi, M. Chaigneau and R. Ossikovski, Chem. Phys. Lett., 2009, 469, 161-165.

18 G. Picardi, M. Chaigneau, R. Ossikovski, C. Licitra and G. Delapierre, J. Raman Spectrosc., 2009, 40, 1407-1412.

19 G. Picardi, A. Kròlikowska, R. Yasukuni, M. Chaigneau, M. Escude, V. Mourier, C. Licitra and R. Ossikovski, chem. phys. chem., 2014, 15, 276-282.

20 M. J. Frisch, G. W. Trucks, H. B. Schlegel, G. E. Scuseria, M. A. Robb, J. R. Cheeseman, G. Scalmani, V. Barone, B. Mennucci, G. A. Petersson, H. Nakatsuji, M. Caricato, X. Li, H. P. Hratchian, A. F. Izmaylov, J. Bloino, G. Zheng, J. L. Sonnenberg, M. Hada, M. Ehara, K. Toyota, R. Fukuda, J. Hasegawa, M. Ishida, T. Nakajima, Y. Honda, O. Kitao, H. Nakai, T. Vreven, J. A. Montgomery, J. E. Peralta, F. Ogliaro, M. Bearpark, J. J. Heyd, E. Brothers, K. N. Kudin, V. N. Staroverov, R. Kobayashi, J. Normand, K. Raghavachari, A. Rendell, J. C. Burant, S. S. Iyengar, J. Tomasi, M. Cossi, N. Rega, J. M. Millam, M. Klene, J. E. Knox, J. B. Cross, V. Bakken, C. Adamo, J. Jaramillo, R. Gomperts, R. E. Stratmann, O. Yazyev, A. J. Austin, R. Cammi, C. Pomelli, J. W. Ochterski, R. L. Martin, K. Morokuma, V. G. Zakrzewski, G. A. Voth, P. Salvador, J. J. Dannenberg, S. Dapprich, A. D. Daniels, Ö. Farkas, J. B. Foresman, J. V. Ortiz, J. Cioslowski and D. J. Fox, 2009, Gaussian, Inc., Wallingford CT.

21 J. P. Perdew, K. Burke and Y. Wang, Phys. Rev. B, 1996, 54, 16533.

22 P. J. Hay and W. R. Wadt, J. Chem. Phys., 1985, 82, 270.

23 M. Sun, Y. Huang, L. Xia, X. Chen and X. Hongxing, J. Phys. Chem. C, 2011, 115, 9629-9636.

24 Lumerical Solut. Inc. http//www.lumerical.com/tcad-products/fdtd/.

25 M.Tabatabaei, A. Sangar, N. Kazemi-Zanjani, P. Torchio, A. Merlen and F. LagugnéLabarthet, J. Phys. Chem. C, 2013, 117, 14778-14786.

26 A. Sangar, A. Merlen, P. Torchio, S. Vedraine, F. Flory, L. Escoubas, L. Patrone, G. Delafosse, V. Chevallier, E. Moyen and M. Hanbucken, Sol. Energy Mater. Sol. Cells, 2013, 117, 657-662.

27 K. Uetsuki, P. Verma, T. Yano, Y. Saito, T. Ichimura and S. Kawata, J. Phys. Chem. C, 2010, 114, 7515-7520.

28 W.-H. Park and Z. H. Kim, Nanoletters, 2010, 10, 4040-4048.

29 L.-B. Zhao, M. Zhang, B. Ren, Z.-Q. Tian and D.-Y. Wu, J. Phys. Chem. C, 2014, 118, 27113-27122.

30 Y. R. Fang, Y. Z. Li, H. X. Xu and M. T. Sun, Langmuir, 2010, 26, 7737-7746. 
31 Y. F. Huang, H. P. Zhu, G. K. Liu, D. Y. Wu, B. Ren and Z. Q. Tian, J. Am. Chem. Soc., 2010, 132, 9244-9246.

32 H. Wang, T. Liu, Y. Huang, Y. Fang, R. Liu, S. Wang, W. Wen and M. Sun, Sci. Rep., 2014, 4, 7087.

33 M. Tabatabaei, A. Sangar, N. Kazemi-Zanjani, P. Torchio, A. Merlen and F. LagugnéLabarthet, J. Phys. Chem. C, 2013, 117, 14778-14786.

34 M. Sun, Z. Zhang, L. Chen, S. S. And and H. Xu, Adv. Opt. Mater., 2014, 2, 74-80.

35 L.-B. Zhao, Y.-F. Huang, X.-M. Liu, J. R. Anema, D.-Y. Wu, B. Ren and Z.-Q. Tian, Phys. Chem. Chem. Phys., 2012, 14, 12919-12929.

36 S. Duan, Y.-J. Ai, W. Hu and Y. Luo, J. Phys. Chem. C, 2014, 118, 6893-6902.

37 L.-B. Zhao, M. Zhang, Y.-F. Huang, C. T. Williams, D.-Y. Wu, B. Ren and Z.-Q. Tian, J. Phys. Chem. Lett., 2014, 5, $1259-1266$.

38 X. Zhang, P. Wang, Z. Zhang, Y. Fang and M. Sun, Sci. Rep., 2014, 4, 5407.

39 L. Cui, P. Wang, X. Chen, Y. Fang, Z. Zhang and M. Sun, Sci. Rep., 2014, 4, 7221.

40 P. Xu, L. Kang, N. H. Mack, K. S. Schanze, X. Han and H.-L. Wang, Sci. Rep., 2013, 3, 2997. 